\title{
CXCLI 2 genetic variants as prognostic markers in nasopharyngeal carcinoma
}

\author{
This article was published in the following Dove Press journal: \\ OncoTargets and Therapy \\ 8 October 2015 \\ Number of times this article has been viewed
}

\author{
Ruiwan Chen ${ }^{1, *}$ \\ Yafei $\mathrm{Xu}^{2, *}$ \\ Xiaojing $\mathrm{Du}^{2, *}$ \\ $\mathrm{Na} \mathrm{L_{iu } { } ^ { 2 }}$ \\ Yingqin $\mathrm{Li}^{2}$ \\ Qingmei $\mathrm{He}^{2}$ \\ Linglong Tang ${ }^{2}$ \\ Yanping $\mathrm{MaO}^{2}$ \\ Ying Sun ${ }^{2}$ \\ Lei Chen ${ }^{2, *}$ \\ Jun $\mathrm{Ma}^{2, *}$
}

'Department of Radiotherapy, The First Affiliated Hospital, Sun Yat-sen University, ${ }^{2}$ Department of Radiation Oncology, State Key Laboratory of Oncology in Southern China, Collaborative Innovation Center of Cancer Medicine, Sun Yat-sen University Cancer Center, Guangzhou, People's Republic of China

*These authors contributed equally to this work
Correspondence: Jun Ma; Lei Chen Department of Radiation Oncology, State Key Laboratory of Oncology in Southern China, Collaborative Innovation Center of Cancer Medicine, Sun Yat-sen University Cancer Center, 65I Dongfeng Road East, Guangzhou 5I0060, People's Republic of China

Tel +862087343469

Fax +862087343295

Email drjunma@hotmail.com; chenlei@sysucc.org.cn
Abstract: The chemokine receptor 4/chemokine ligand 12 (CXCR4/CXCL12) axis plays an important role in tumorigenesis, metastasis, and recurrence of tumors. Its single nucleotide polymorphisms (SNPs) are associated with patient survival in several types of cancer. However, the prognostic value of SNPs in nasopharyngeal carcinoma (NPC) has not been fully investigated. This retrospective study assessed the relationships between CXCR4 rs2228014 and CXCL12 rs1801157 polymorphisms and patient outcome in 222 patients newly diagnosed with NPC. The analysis found no significant correlation between the presence of both SNPs and clinicopathological factors. However, univariate analysis showed that $\mathrm{N}$ classification, clinical stage, and the CXCL12 rs1801157 polymorphism were significantly associated with distant metastasis-free survival ( $P=0.018,0.028$, and 0.013 , respectively) and progression-free survival ( $P=0.007,0.046$, and 0.021 , respectively). After adjusting clinicopathological factors, multivariate analysis identified CXCL12 rs1801157 as an independent prognostic factor for distant metastasis-free survival and progression-free survival (hazard ratio: 3.332; 95\% confidence interval: $1.597-6.949 ; P=0.001$ and hazard ratio: $2.66595 \%$ confidence interval: $1.387-5.119$; $P=0.003$, respectively). Our results suggest that $C X C L 12$ rs 1801157 AA genotype might serve as a potential prognostic factor in patients with NPC.

Keywords: nasopharyngeal carcinoma, $C X C R 4, C X C L 12$, polymorphism, prognosis

\section{Introduction}

Nasopharyngeal carcinoma (NPC) is the most common nasopharyngeal malignancy, with an extremely unbalanced endemic distribution. According to the International Agency for Research on Cancer, 84,400 cases of NPC were newly diagnosed in 2008, with 51,600 mortalities worldwide. Approximately $40 \%$ of these occurred in the People's Republic of China. ${ }^{1}$ Despite combined applications of magnetic resonance imaging (MRI), intensity-modulated radiation therapy, and chemotherapy, distant metastasis occurs in $20 \%-30 \%$ of patients and is the main cause of treatment failure in NPC. ${ }^{2}$

Tumor, node, metastasis (TNM) classification is widely used for prognosis evaluation and establishment of treatment strategy. However, due to the heterogeneity of the tumor in NPC, patients at the same TNM stage who are undergoing similar treatments frequently experience different clinical outcomes, suggesting that the TNM staging system is inadequate for prognostic stratification in NPC. ${ }^{3}$ Therefore, the development of new prognostic markers is required as an adjunct to traditional staging techniques in order to develop patient-specific treatment strategies for patients with NPC.

It is generally recognized that chemokines and their receptors play an important role in the metastatic process by guiding the migration of receptor-bearing tumor cells to secondary tissues where their ligands are expressed. ${ }^{4}$ Chemokine receptor 4 (CXCR4) is widely expressed in malignant cells, and its sole ligand is chemokine 
ligand 12 (CXCL12). ${ }^{5}$ The CXCR4/CXCL12 axis facilitates tumor metastasis by directly guiding tumor cells to metastatic sites. As such, it has been implicated in promoting growth, proliferation, and angiogenesis of tumor cells. ${ }^{6}$ Increased expression of CXCR4 and CXCL12 has been associated with poor prognosis in several tumors. ${ }^{7,8}$

The CXCR4 gene is located on chromosome $2 \mathrm{q} 2^{9}$ with a silent single nucleotide polymorphism (SNP) at codon 138, named rs2228014. ${ }^{10}$ The CXCL12 gene is located on chromosome 10q11.1, ${ }^{11}$ with a functional SNP rs 1801157 in the 3 '-untranslated gene region. ${ }^{12}$ Both polymorphisms have been associated with an increased risk of cancer and poorer prognoses in colorectal, ${ }^{13}$ endometrial, ${ }^{14}$ and lung cancer. ${ }^{15}$ However, little is known about the prognostic value of these two polymorphisms in NPC.

Genetic background may contribute to the differences in the level and activities of CXCR 4 and CXCL12 between patients. To explore the hypothesis that CXCR4 rs2228014 and CXCL12 rs1801157 polymorphisms may be associated with clinical outcome in patients with NPC, we retrospectively investigated the relationship between these two polymorphisms and the clinicopathological characteristics of 222 untreated patients with NPC from a single endemic area in the People's Republic of China and their association with prognosis.

\section{Materials and methods}

\section{Patient selection, treatment, and follow-up}

A total of 222 patients, diagnosed with NPC at the Sun Yat-sen University Cancer Center (Guangzhou, People's Republic of China) between December 2000 and March 2007, were enrolled in this study. The selection criteria were as follows: ethnic Han Chinese; newly diagnosed with histologically confirmed NPC and with no distant metastasis; no history of any other malignant disease; Karnofsky score $\geq 70$; no prior treatment for NPC; treated with radiotherapy at our Cancer Center; underwent regular follow-ups; and availability of periphery blood samples. All medical records were reviewed retrospectively, and all patients were restaged according to the seventh edition of the UICC/AJCC TNM classification system. Written informed consents had been given by all patients, and the research was approved by the Center's ethics committee.

Before enrollment, each patient underwent a complete pretreatment evaluation, including patient history, physical examinations, hematological and biochemical profiling, MRI of the head and neck, chest radiography, abdominal sonography, and whole-body bone scan. All patients received definitive radiotherapy following established methods. ${ }^{16}$
The accumulated dose to the primary tumor was 64-80 Gy; the accumulated dose to the involved areas of the neck was 60-62 Gy, and it was 50 Gy to the uninvolved areas. Concurrent chemotherapy consisted of cisplatin administered weekly or on weeks 1, 4, and 7 of radiotherapy. Neoadjuvant and adjuvant chemotherapy consisted of cisplatin with 5-fluorouracil or taxanes administered every 3 weeks for three cycles.

Follow-up was carried out every 3 months during the first year and every 6 months thereafter, until the final follow-up or death. Follow-up was concluded on May 2015. The median follow-up period was 86.6 months (range: 3.1-152.9 months). Distant metastasis-free survival (DMFS) and progressionfree survival (PFS) were selected as the endpoints of this study. DMFS was calculated from the date of enrollment to the date of distant metastasis or the last follow-up. PFS was calculated from the date of enrollment to the date of any form of tumor progression or to the last follow-up.

\section{DNA extraction and genotyping}

Peripheral blood $(2 \mathrm{~mL})$ was collected at the time of enrollment for genotyping. DNA was obtained from peripheral lymphocytes using the QIAamp DNA Blood Midi Kit (QIAGEN; Valencia, CA, USA) according to the manufacturer's instructions. The primers for the polymerase chain reaction (PCR) analysis of CXCR4 and CXCL12 polymorphisms were designed using Primer 5.0 software and synthesized commercially by BGI (Shenzhen, People's Republic of China). The primer sequences were as follows: CXCR4 rs2228014; forward: 5'-TCCGTGAAGAAAATGCTAAT-3'; reverse: 5'-AACACAACCACCCACAAGTC-3' and CXCL12 rs 1801157; forward: 5'-TCTTTCCACGGAGCCACT-3'; reverse: 5'-TCATTTCCTGCTTGGTGC-3'.

PCR amplification was performed in a $30 \mu \mathrm{L}$ reaction volume containing $2 \mu \mathrm{L}$ genomic DNA, $2 \mu \mathrm{L} 2.5$ mM dNTPs, $3 \mu \mathrm{L} 10 \times$ rTaq buffer, $1 \mu \mathrm{L}$ of each primer $(10 \mathrm{mM})$, and $0.2 \mu \mathrm{L}$ rTaq. PCRs were performed under the following conditions: initial denaturation at $94^{\circ} \mathrm{C}$ for 5 minutes, followed by 30 cycles of 20 seconds at $95^{\circ} \mathrm{C}$, annealing at $65^{\circ} \mathrm{C}$ for 20 seconds, polymerization at $72^{\circ} \mathrm{C}$ for 30 seconds, with a final polymerization step at $72^{\circ} \mathrm{C}$ for 5 minutes. The PCR products were purified and sequenced by BGI, and the sequence data were analyzed using DNASTAR analysis programs.

\section{Statistical analysis}

All the analyses, with the exception of the Schoenfeld residual plot test, were performed using v17.0 software (SPSS Inc., Chicago, IL, USA). Pearson's chi-squared test $\left(\chi^{2}\right)$ was 
used to determine correlations between the two SNPs and the patients' clinicopathological features. The $\chi^{2}$ test was also used to test the Hardy-Weinberg equilibrium. The KaplanMeier method was used to estimate the actuarial rates, and the survival curves were compared by the log-rank test. Schoenfeld residual plot test was applied to test the proportional hazard assumption, using the Stata statistical software (STATA 12; StataCorp LP, College Station, TX, USA).

Univariate and multivariate analyses were performed using a Cox proportional hazards model in order to calculate hazard ratios (HRs) and determine the independent significances of explanatory variables by backward elimination. The following parameters were selected as the covariates in the multivariate analysis: age, sex, smoking status, alcohol consumption, histological type, radiotherapy technique, chemotherapy, clinical stage, and CXCL12 rs1801157. Two-tailed $P$-values $<0.05$ were considered statistically significant.

\section{Results}

\section{Patient characteristics and clinical outcomes}

The distribution of the clinicopathological features of the patients are summarized in Table 1. The median age was 48 years (range: $14-80$ years). Two-dimensional radiotherapy was administered to 208/222 (93.7\%) patients, and intensity-modulated radiation therapy was administered to 14/222 (6.3\%) patients. A total of 150/222 (67.6\%) patients received chemotherapy. The distribution of clinical stages was similar to those observed in other studies performed in our cancer center. At the final follow-up, 54 patients (24.3\%) had died, and the 5-year DMFS and PFS rates were $82.8 \%$ and $74.4 \%$, respectively.

\section{Correlation of CXCR4 and CXCLI 2 polymorphisms with patients' clinicopathological features}

Genotyping showed that the distribution of the CXCR4 rs2228014 genotype within the 222 enrolled patients was $70.3 \%$ (149/222) CC (homozygous wild allele), 25.5\% (54/222) CT, and 4.2\% (9/222) TT, respectively. The distribution of CXCL12 rs1801157 genotype was 51.9\% (109/222) GG (homozygous wild allele), 39.1\% (82/222) GA, and 9.0\% (19/222) AA. The completion rates of these two polymorphisms were $94.6 \%$ and $95.5 \%$ for CXCR4 rs 2228014 and CXCL12 rs 1801157, respectively. Representative sequencing chromatograms from randomly chosen cases were used to illustrate these distributions (Figure 1).
Table I Clinicopathological characteristics of patients with NPC who were enrolled in this study $(n=222)$

\begin{tabular}{|c|c|}
\hline Characteristic & $\begin{array}{l}\text { Number of } \\
\text { patients (\%) }\end{array}$ \\
\hline \multicolumn{2}{|l|}{ Age } \\
\hline$\geq 48$ years & II 5 (5I.8) \\
\hline$<48$ years & $107(48.2)$ \\
\hline \multicolumn{2}{|l|}{ Sex } \\
\hline Male & I 75 (78.8) \\
\hline Female & $47(2 \mid .2)$ \\
\hline \multicolumn{2}{|l|}{ Smoking status } \\
\hline Ever & $103(46.4)$ \\
\hline Never & $119(53.6)$ \\
\hline \multicolumn{2}{|c|}{ Alcohol consumption } \\
\hline Ever & $49(22.1)$ \\
\hline Never & $173(77.9)$ \\
\hline \multicolumn{2}{|l|}{ Histology } \\
\hline WHO I type & $\mathrm{I}(0.5)$ \\
\hline WHO II type & $12(5.4)$ \\
\hline WHO III type & $209(94.1)$ \\
\hline \multicolumn{2}{|l|}{ Radiotherapy } \\
\hline IMRT & $14(6.3)$ \\
\hline 2D-RT & $208(93.7)$ \\
\hline \multicolumn{2}{|l|}{ Chemotherapy } \\
\hline Yes & $150(67.6)$ \\
\hline No & $72(32.4)$ \\
\hline \multicolumn{2}{|l|}{ T classification } \\
\hline $\mathrm{T}_{1-2}$ & $93(4 I .9)$ \\
\hline $\mathrm{T}_{3-4}$ & $129(68.1)$ \\
\hline \multicolumn{2}{|l|}{$\mathrm{N}$ classification } \\
\hline$N_{1-2}$ & $170(76.6)$ \\
\hline $\mathrm{N}_{3-4}^{-2}$ & $52(23.4)$ \\
\hline \multicolumn{2}{|l|}{ Clinical stage } \\
\hline I-II & $79(35.6)$ \\
\hline III-IV & $143(64.4)$ \\
\hline
\end{tabular}

Abbreviations: NPC, nasopharyngeal carcinoma; WHO, World Health Organization; IMRT, intensity-modulated radiotherapy; 2D-RT, two-dimensional radiotherapy.

Although the genotype frequencies for these two polymorphisms were confirmed to be in Hardy-Weinberg equilibrium for patients with NPC, there were no statistically significant associations between genotypes and sex, age, smoking status, alcohol consumption, histology, radiotherapy technique, chemotherapy, $\mathrm{T}$ classification, $\mathrm{N}$ classification, or clinical stage (data not shown).

\section{Univariate analysis}

In relation to patients' clinicopathological features, DMFS (HR: $1.034 ; 95 \%$ confidence interval [CI]: 1.006-1.064; $P=0.018$ and HR: 1.037 ; 95\% CI: $1.004-1.071 ; P=0.028$, respectively) and PFS (HR: 1.032; 95\% CI: 1.009-1.057; $P=0.007$ and HR: 1.026 ; $95 \%$ CI: $1.000-1.052 ; P=0.046$, respectively) were both significantly associated with $\mathrm{N}$ classification and clinical stage, as expected. In contrast, none of the other tested characteristics (age, sex, smoking status, alcohol consumption, histological type, radiotherapy 
A

CC

300

T G G C C A T C G T C C A C G

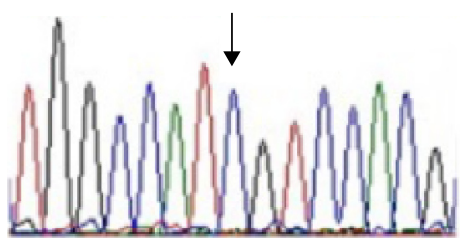

D GG

$220 \quad 230$

G G G A G C C G G G T C T G C C

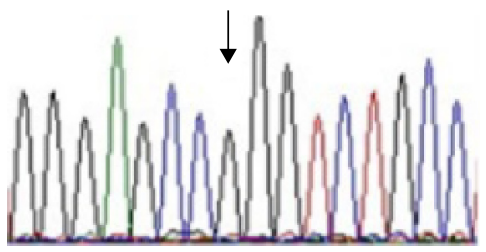

B

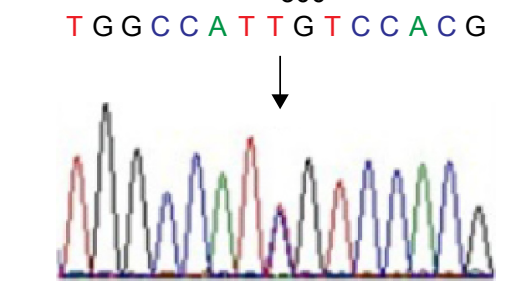

E

0

G G G A G C C G G G T C T G C C

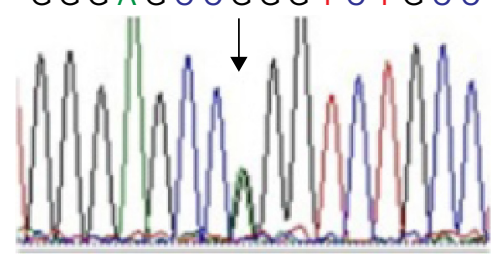

C

TT

290

300
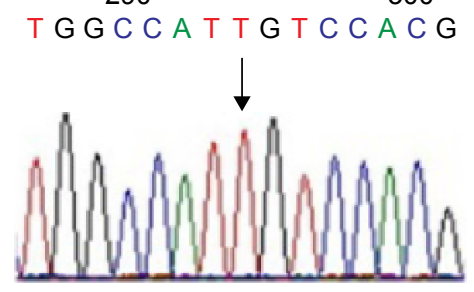

$\mathbf{F}$

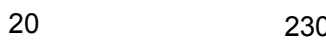

G G G A G C C A G G T C T G C C

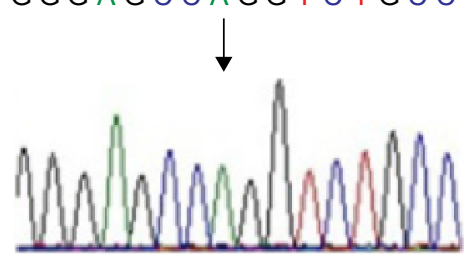

Figure I Sequencing chromatograms of CXCR4 rs2228014 and CXCLI2 rs 1801157.

Notes: (A-C) Shows the chromatograms for CXCR4 rs22280 I 4 CC, CT, and TT genotypes, respectively. (D-F) Shows the chromatograms for CXCLI2 rs 180 I I57 GG, GA, and AA genotypes, respectively. The results represent randomly selected samples. Abbreviations: CXCR4, chemokine receptor 4; CXCLI2, chemokine ligand I2.

technique, chemotherapy, and T classification) has a significant effect on either DMFS or PFS.

In relation to genotype, univariate analysis found no significant association between $C X C R 4$ genotypes and DMFS or PFS ( $P=0.998, P=0.833$, respectively; Table 2 ). In contrast, a close association was found between $C X C L 12$ GG and GA genotypes and improved prognosis, compared to patients carrying the $C X C L 12$ AA genotype ( $P=0.044$ and $P=0.062$ for DMFS and PFS, respectively; Table 2, Figure $2 \mathrm{~A}$ and $\mathrm{C}$ ). The significance of this association was increased when patients carrying $G$ alleles $(G G+$ GA) were combined for further analysis, compared to patients carrying the $C X C L 12$ AA genotype ( $P=0.013$ and $P=0.021$ for DMFS and PFS, respectively; Table 2, Figure $2 \mathrm{~B}$ and $\mathrm{D})$.

\section{Multivariate analysis}

After adjusting for clinicopathological features (age, sex, smoking status, alcohol consumption, histological type, radiotherapy technique, chemotherapy, and clinical stage), multivariate analysis using Cox proportional hazards model confirmed that the CXCL12 rs 1801157 polymorphism was a significant independent prognostic factors for DMFS (HR: 3.332; 95\% CI: 1.597-6.949; $P=0.001$ ) and PFS (HR: 2.665 ; 95\% CI: $1.387-5.119 ; P=0.003$ ) in patients with NPC (Table 3). Clinical stage was also identified as an independent prognostic factor for DMFS (HR: 2.873;
95\% CI: 1.378-5.988; $P=0.005)$ and PFS (HR: $1.806 ; 95 \%$ CI: $1.033-3.158 ; P=0.038$; Table 3 ).

\section{Discussion}

To date, there has been considerable research in identifying new biomarkers for more accurate prognostic assessment in NPC. Tumor-derived markers, including Epstein-Barr virus DNA,${ }^{17}$ VEGF, ${ }^{18}$ and IL- $8{ }^{19}$ have been proposed as potential candidates. Germline polymorphism is a common approach to explore the value of host-derived factors. The advantages of SNP genotyping, compared to gene expression profiling, include less variability between laboratories and across platforms and the ability to analyze both tissue samples and peripheral blood. ${ }^{20}$

CXCL12 is believed to attract tumor cells by binding to its sole receptor CXCR4, which is widely expressed in tumor cells. ${ }^{5}$ The CXCR4/CXCL12 axis has multiple functions in tumorigenesis, including tumor growth, angiogenesis, distant metastasis, and survival. ${ }^{6}$ However, the prognostic value of CXCR4 and CXCL12 genetic variants in patients with NPC is unclear. Therefore, the aim of this study was to investigate the association between two specific $C X C R 4$ and CXCL12 genetic polymorphisms, namely CXCR4 rs 2228014 and CXCL12 rs1801157, and the clinical outcomes of 222 patients with NPC in a single endemic area.

Activation of CXCR4/CXCL12 in the tumor microenvironment has been implicated in tumor growth and 
Table 2 Univariate analysis of DMFS and PFS in patients with NPC $(n=222)$

\begin{tabular}{|c|c|c|c|c|c|c|}
\hline \multirow[t]{2}{*}{ Variable } & \multicolumn{3}{|l|}{ DMFS } & \multicolumn{3}{|l|}{ PFS } \\
\hline & HR & $95 \% \mathrm{Cl}$ & $P$-value & HR & $95 \% \mathrm{Cl}$ & $P$-value \\
\hline Age & & & 0.290 & & & 0.342 \\
\hline$<48$ years & 1.000 & & & 1.000 & & \\
\hline$\geq 48$ years & 1.384 & $0.758-2.526$ & & 1.269 & $0.776-2.075$ & \\
\hline Sex & & & 0.360 & & & 0.110 \\
\hline Male & 1.000 & & & 1.000 & & \\
\hline Female & 1.459 & $0.650-3.274$ & & 1.776 & $0.879-3.592$ & \\
\hline Smoking status & & & 0.875 & & & 0.959 \\
\hline Never & 1.000 & & & 1.000 & & \\
\hline Ever & 1.046 & $0.586-1.837$ & & 0.988 & $0.624-1.565$ & \\
\hline Alcohol consumption & & & 0.656 & & & 0.646 \\
\hline Never & 1.000 & & & 1.000 & & \\
\hline Ever & 0.851 & $0.413-1.754$ & & 1.137 & $0.66 \mathrm{I}-1.957$ & \\
\hline Histological type & & & 0.743 & & & 0.631 \\
\hline WHO I & 1.000 & & & 1.000 & & 0.631 \\
\hline WHO II & $4,416.604$ & $0.000-8.29 E 89$ & & $4,44 I .070$ & $0.000-7.438$ E73 & \\
\hline WHO III & $2,956.944$ & $0.000-5.538 \mathrm{E} 89$ & & $2,955.821$ & $0.000-4.94$ IE73 & \\
\hline Radiotherapy & & & 0.523 & & & 0.417 \\
\hline 2D-RT & 1.000 & & & 1.000 & & \\
\hline IMRT & 0.630 & $0.152-2.604$ & & 0.619 & $0.194-1.972$ & \\
\hline Chemotherapy & & & 0.280 & & & 0.951 \\
\hline No & 1.000 & & & 1.000 & & \\
\hline Yes & 1.444 & $0.74|-2.8| 3$ & & 0.984 & $0.587-1.648$ & \\
\hline T classification & & & 0.425 & & & 0.572 \\
\hline $\mathrm{T}_{1-2}$ & 1.000 & & & 1.000 & & \\
\hline $\mathrm{T}_{3-4}$ & 1.011 & $0.984-1.040$ & & 1.007 & $0.984-1.030$ & \\
\hline $\mathrm{N}$ classification & & & 0.018 & & & 0.007 \\
\hline $\mathrm{N}_{0-1}$ & 1.000 & & & 1.000 & & \\
\hline $\mathrm{N}_{2-3}$ & 1.034 & $1.006-1.064$ & & 1.032 & $1.009-1.057$ & \\
\hline Clinical stage & & & 0.028 & & & 0.046 \\
\hline I-II & 1.000 & & & 1.000 & & \\
\hline III-IV & 1.037 & $|.004-1.07|$ & & 1.026 & $1.000-1.052$ & \\
\hline CXCR4 rs $22280 I 4$ & & & 0.998 & & & 0.833 \\
\hline $\mathrm{TT}$ & 1.000 & & & 1.000 & & \\
\hline $\mathrm{CT}$ & 0.955 & $0.229-3.980$ & & 1.507 & $0.367-6.194$ & \\
\hline $\mathrm{CC}$ & 0.958 & $0.214-4.285$ & & 1.403 & $0.324-6.075$ & \\
\hline CXCLI2 rs 1801157 & & & 0.044 & & & 0.062 \\
\hline $\mathrm{AA}$ & 1.000 & & & 1.000 & & \\
\hline GA & 0.384 & $0.171-0.864$ & & 0.425 & $0.208-0.87 \mid$ & \\
\hline \multirow[t]{2}{*}{ GG } & 0.370 & $0.158-0.866$ & & 0.486 & $0.234-1.008$ & \\
\hline & & & 0.013 & & & 0.021 \\
\hline $\mathrm{GA}+\mathrm{GG}$ & 0.378 & $0.176-0.811$ & & 0.451 & $0.230-0.885$ & \\
\hline
\end{tabular}

Note: $P<0.05$ was considered statistically significant.

Abbreviations: DMFS, distant metastasis-free survival; PFS, progression-free survival; NPC, nasopharyngeal carcinoma; HR, hazard ratio; Cl, confidence interval; WHO, World Health Organization; 2D-RT, two-dimensional radiotherapy; IMRT, intensity-modulated radiotherapy; T, tumor; N, node; CXCLI2, chemokine ligand I2; CXCR4, chemokine receptor 4 .

distant metastasis. ${ }^{21,22}$ In vitro and in vivo studies have been supported by retrospective studies showing a close relationship between expression of CXCR 4 and CXCL12 and disease stage and prognosis in several malignancies, including melanoma, ${ }^{23}$ prostate cancer, ${ }^{24}$ and pancreatic cancer. ${ }^{25}$ Although high expression of CXCR4 has been linked to metastatic progression and poor prognosis in patients with NPC, ${ }^{8,26}$ details of the role and molecular mechanisms of the CXCR4/CXCL12 axis in NPC remains scarce.

Differences in genetic polymorphisms may contribute to the variability in CXCR4 and CXCL12 expression between individuals. A significant association between CXCL12 rs1801157 genotypes and susceptibility and prognosis in breast cancer and several other malignancies have been reported. ${ }^{14,27}$ However, the influence of the CXCL12 
A

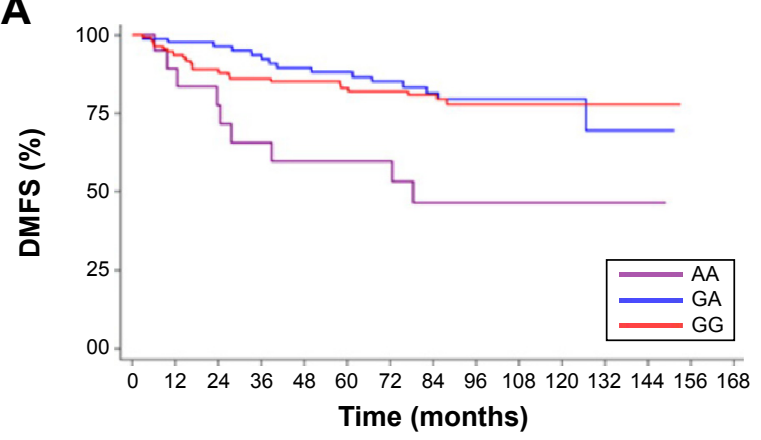

B

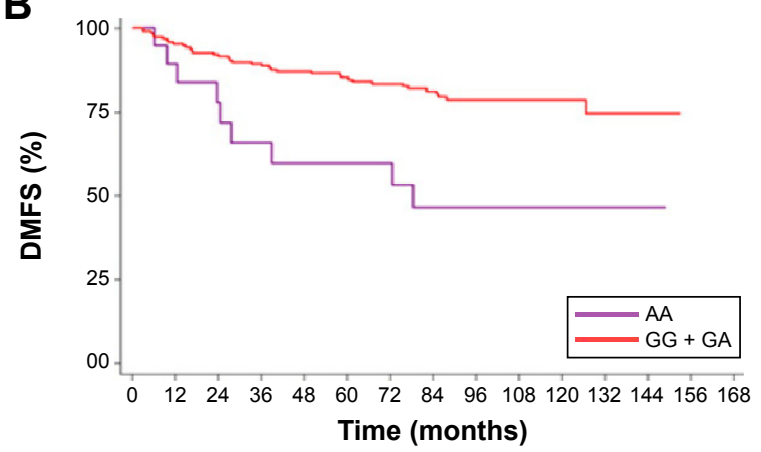

C

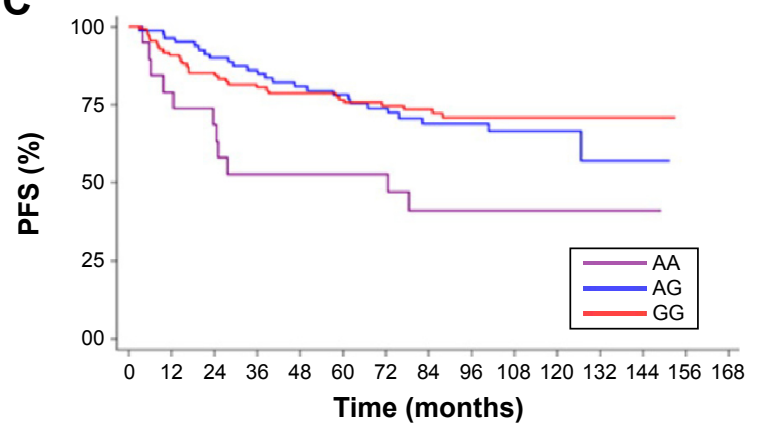

D

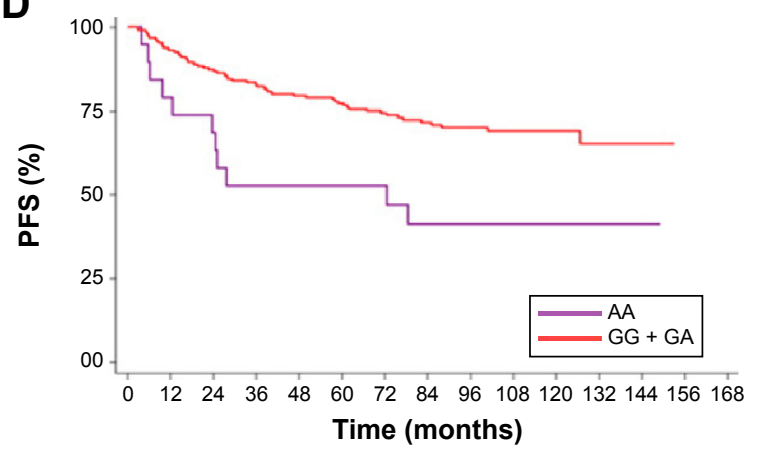

Figure 2 Kaplan-Meier curves of DMFS and PFS for the different CXCLI 2 rs I80II 57 genotypes.

Notes: (A and C) Compare GG, GA, and AA genotypes for DMFS and PFS. (B and D) Compare GA + GG and AA genotypes for DMFS and PFS. P-value was calculated by log-rank test.

Abbreviations: CXCLI2, chemokine ligand I2; DMFS, distant metastasis-free survival; PFS, progression-free survival.

rs 1801157 polymorphism in the expression of the CXCL12 protein remains controversial. Studies have shown that CXCL12 rs1801157 homozygous AA genotype was correlated with the increased levels of protein expression, ${ }^{12,27}$ a higher risk of cancer, and poorer clinical outcome in several malignancies. However, a conflicting result was found in breast cancer, with significantly lower expression levels of CXCL12 mRNA in peripheral blood samples in patients carrying CXCL12 rs 1801157 A alleles compared to those with the GG alleles. ${ }^{28}$ Consequently, further research is required to determine the molecular mechanisms that underline CXCL12 rs1801157 polymorphism and gene expression.

Table 3 Multivariate analysis for DMFS and PFS in patients with NPC ( $n=222)$

\begin{tabular}{lllll}
\hline Endpoint & Variable & HR & $\mathbf{9 5 \%} \mathbf{C l}$ & P-value \\
\hline DMFS & Clinical stage & 2.873 & $1.378-5.988$ & 0.005 \\
& $C X C L I 2$ rsI80II57 & 3.332 & $1.597-6.949$ & 0.001 \\
PFS & Clinical stage & 1.806 & $1.033-3.158$ & 0.038 \\
& $C X C L I 2$ rs I80II57 & 2.665 & $1.387-5.119$ & 0.003 \\
\hline
\end{tabular}

Note: $P<0.05$ was considered statistically significant.

Abbreviations: DMFS, distant metastasis-free survival; PFS, progression-free survival; NPC, nasopharyngeal carcinoma; $\mathrm{HR}$, hazard ratio; $\mathrm{Cl}$, confidence interval; $C X C L / 2$, chemokine ligand 12 .
Our study reveals that the CXCL12 rs1801157 AA genotype was significantly associated with poor DMFS and PFS compared to GG and GA genotype, which are consistent with recent studies of other malignancies. CXCL12 AA genotype and $C X C R 4$ TT genotype contributed to higher odds ratio for lung cancer, and patients carrying CXCL12 AA or CXCR4 TT genotype exhibited a tendency to advanced cancer stage and poorer prognosis compared with those with other genotypes. ${ }^{15}$ Similar findings have been reported in colorectal cancer and esophagogastric cancer. ${ }^{29}$ We hypothesized that the $\mathrm{G} \rightarrow \mathrm{A}$ mutation in CXCL12 rs1801157 could alter gene expression, thereby influencing the migration and metastasis in NPC. The earlier studies indicate that CXCL12 rs1801157 has great potential as a novel prognostic biomarker in several malignancies, and the critical effect of CXCL12 in NPC progression is of great value for further exploration.

CXCR4 rs2228014 is a silent SNP. Patients carrying $\mathrm{T}$ allele have been linked to higher cancer risk and poorer prognosis in endometrial carcinoma and non-small-cell lung cancer than those carrying $\mathrm{C}$ alleles. ${ }^{14,15}$ However, our results found no significant relationship between the CXCR4 rs2228014 genotypes and prognosis in NPC. It has been suggested that this silent SNP may be linked to an as yet 
unknown functional mutation that can modify gene expression, induce differences in transfer RNA (tRNA) selection, and alter RNA stability, folding, or splicing. ${ }^{30}$

The CXCR4/CXCL12 axis is attracting increasing interest as a potential target for the development of anticancer therapeutics. ${ }^{31}$ To date, over 15 new drugs targeting the CXCR4/CXCL12 axis have been developed..$^{32}$ AMD3100, a CXCR4 antagonist also known as plerixafor (Mozobil, Sanofi US, Bridgewater, NJ, USA), has been approved for use in patients with non-Hodgkin's lymphoma and multiple myeloma, by the Food and Drug Administration. ${ }^{33,34}$ Phase I and II clinical trials have been carried out on other drugs, including TG-0054, a CXCR4 inhibitor, and CTCE-9908, ${ }^{35}$ a CXCL12 peptide analog. These developments promote the therapeutic potential of the CXCR4/CXCL12 axis against NPC.

\section{Conclusion}

This study has demonstrated that the CXCL12 rs1801157 genotype was significantly associated with poorer DMFS and PFS in patients with NPC, compared to CXCL12 rs1801157 GG and GA genotypes. This suggests that the inhibition of the CXCR4/CXCL12 signaling pathway may provide a novel therapeutic strategy for the treatment of patients with NPC. The study had the following limitations: the cohort was small, so the results will need to be validated using larger sample sizes, and the details of the underlying mechanisms were not investigated. Despite these limitations, the close association between the CXCL12 rs1801157 polymorphism and prognosis in patients with NPC warrants further investigation and has demonstrated its potential as a promising therapeutic target in NPC.

\section{Acknowledgments}

This work was supported by grants from the Guangdong Province Universities and Colleges Pearl River Scholar Funded Scheme (2010), the Innovation Team Development Plan of the Ministry of Education (number IRT1297), the National Natural Science Foundation of China (number 81302366, 81230056), and the Science and Technology Project of Guangzhou (number 12BppZXaa2060002).

\section{Disclosure}

The authors report no conflicts of interest in this work.

\section{References}

1. Jemal A, Bray F, Center MM, Ferlay J, Ward E, Forman D. Global cancer statistics. CA Cancer J Clin. 2011;61:69-90.

2. Kam MK, Teo PM, Chau RM, et al. Treatment of nasopharyngeal carcinoma with intensity-modulated radiotherapy: the Hong Kong experience. Int J Radiat Oncol Biol Phys. 2004;60:1440-1450.
3. Chen L, Mao YP, Xie FY, et al. The seventh edition of the UICC/ AJCC staging system for nasopharyngeal carcinoma is prognostically useful for patients treated with intensity-modulated radiotherapy from an endemic area in China. Radiother Oncol. 2012;104:331-337.

4. Mantovani A, Allavena P, Sica A, Balkwill F. Cancer-related inflammation. Nature. 2008;454:436-444.

5. Burger JA, Kipps TJ. CXCR4: a key receptor in the crosstalk between tumor cells and their microenvironment. Blood. 2006;107: 1761-1767.

6. Teicher BA, Fricker SP. CXCL12 (SDF-1)/CXCR4 pathway in cancer. Clin Cancer Res. 2010;16:2927-2931.

7. Saigusa S, Toiyama Y, Tanaka K, et al. Stromal CXCR4 and CXCL12 expression is associated with distant recurrence and poor prognosis in rectal cancer after chemoradiotherapy. Ann Surg Oncol. 2010;17: 2051-2058.

8. Wang N, Wu QL, Fang Y, et al. Expression of chemokine receptor CXCR4 in nasopharyngeal carcinoma: pattern of expression and correlation with clinical outcome. J Transl Med. 2005;3:26.

9. Federsppiel B, Melhado IG, Duncan AM, et al. Molecular cloning of the cDNA and chromosomal localization of the gene for a putative seven-transmembrane segment (7-TMS) receptor isolated from human spleen. Genomics. 1993;16:707-712.

10. Petersen DC, Glashoff RH, Shrestha S, et al. Risk for HIV-1 infection associated with a common CXCL12 (SDF1) polymorphism and CXCR4 variation in an African population. J Acquir Immune Defic Syndr. 2005;40:521-526.

11. Shirozu M, Nakano T, Inazawa J, et al. Structure and chromosomal localization of the human stromal cell-derived factor 1 (SDF1) gene. Genomics. 1995;28:495-500.

12. Winkler C, Modi W, Smith MW, et al. Genetic restriction of AIDS pathogenesis by an SDF-1 chemokine gene variant. ALIVE Study, Hemophilia Growth and Development Study (HGDS), Multicenter AIDS Cohort Study (MACS), Multicenter Hemophilia Cohort Study (MHCS), San Francisco City Cohort (SFCC). Science. 1998;279: 389-393.

13. Chang SC, Lin PC, Yang SH, Wang HS, Li AF, Lin JK. SDF-1alpha G801A polymorphism predicts lymph node metastasis in stage T3 colorectal cancer. Ann Surg Oncol. 2009;16:2323-2330.

14. Cacina C, Bulgurcuoglu-Kuran S, Iyibozkurt AC, Yaylim-Eraltan I, Cakmakoglu B. Genetic variants of SDF-1 and CXCR4 genes in endometrial carcinoma. Mol Biol Rep. 2012;39:1225-1229.

15. Lee YL, Kuo WH, Lin CW, et al. Association of genetic polymorphisms of CXCL12/SDF1 gene and its receptor, CXCR4, to the susceptibility and prognosis of non-small cell lung cancer. Lung Cancer. 2011; 73:147-152.

16. Lai SZ, Li WF, Chen L, et al. How does intensity-modulated radiotherapy versus conventional two-dimensional radiotherapy influence the treatment results in nasopharyngeal carcinoma patients? Int J Radiat Oncol Biol Phys. 2011;80:661-668.

17. Leung SF, Zee B, Ma BB, et al. Plasma Epstein-Barr viral deoxyribonucleic acid quantitation complements tumor-node-metastasis staging prognostication in nasopharyngeal carcinoma. J Clin Oncol. 2006; 24:5414-5418.

18. Lv X, Xiang YQ, Cao SM, et al. Prospective validation of the prognostic value of elevated serum vascular endothelial growth factor in patients with nasopharyngeal carcinoma: more distant metastases and shorter overall survival after treatment. Head Neck. 2011;33:780-785.

19. Li XJ, Peng LX, Shao JY, et al. As an independent unfavorable prognostic factor, IL-8 promotes metastasis of nasopharyngeal carcinoma through induction of epithelial-mesenchymal transition and activation of AKT signaling. Carcinogenesis. 2012;33:1302-1309.

20. Hunter KW, Crawford NP. Germ line polymorphism in metastatic progression. Cancer Res. 2006;66:1251-1254.

21. Taichman RS, Cooper C, Keller ET, Pienta KJ, Taichman NS, McCauley LK. Use of the stromal cell-derived factor-1/CXCR4 pathway in prostate cancer metastasis to bone. Cancer Res. 2002;62:1832-1837. 
22. Sun YX, Schneider A, Jung Y, et al. Skeletal localization and neutralization of the SDF-1 (CXCL12)/CXCR4 axis blocks prostate cancer metastasis and growth in osseous sites in vivo. J Bone Miner Res. 2005; 20:318-329.

23. Scala S, Ottaiano A, Ascierto PA, et al. Expression of CXCR4 predicts poor prognosis in patients with malignant melanoma. Clin Cancer Res. 2005;11:1835-1841.

24. Akashi T, Koizumi K, Tsuneyama K, Saiki I, Takano Y, Fuse H. Chemokine receptor CXCR4 expression and prognosis in patients with metastatic prostate cancer. Cancer Sci. 2008;99:539-542.

25. Liang JJ, Zhu S, Bruggeman R, et al. High levels of expression of human stromal cell-derived factor-1 are associated with worse prognosis in patients with stage II pancreatic ductal adenocarcinoma. Cancer Epidemiol Biomarkers Prev. 2010;19:2598-2604.

26. Segawa Y, Oda Y, Yamamoto H, et al. Close correlation between CXCR4 and VEGF expression and their prognostic implications in nasopharyngeal carcinoma. Oncol Rep. 2009;21:1197-1202.

27. Hirata H, Hinoda Y, Kikuno N, et al. CXCL12 G801A polymorphism is a risk factor for sporadic prostate cancer susceptibility. Clin Cancer Res. 2007;13:5056-5062.

28. de Oliveira KB, Guembarovski RL, Oda JM, et al. CXCL12 rs 1801157 polymorphism and expression in peripheral blood from breast cancer patients. Cytokine. 2011;55:260-265.
29. Schimanski CC, Jordan M, Schlaegel F, et al. SNP rs1801157 significantly correlates with distant metastasis in CXCL12 expressing esophagogastric cancer. Int J Oncol. 2011;39:515-520.

30. Shastry BS. SNPs: impact on gene function and phenotype. Methods Mol Biol. 2009;578:3-22.

31. Albert S, Riveiro ME, Halimi C, et al. Focus on the role of the CXCL12/ CXCR4 chemokine axis in head and neck squamous cell carcinoma. Head Neck. 2013;35(12):1819-1828.

32. Peled A, Wald O, Burger J. Development of novel CXCR4-based therapeutics. Expert Opin Investig Drugs. 2012;21:341-353.

33. DiPersio JF, Stadtmauer EA, Nademanee A, et al. Plerixafor and G-CSF versus placebo and G-CSF to mobilize hematopoietic stem cells for autologous stem cell transplantation in patients with multiple myeloma. Blood. 2009;113:5720-5726.

34. De Clercq E. The AMD3100 story: the path to the discovery of a stem cell mobilizer (Mozobil). Biochem Pharmacol. 2009;77:1655-1664.

35. Hotte SJ, Hirte HW, Moretto P, et al. Final results of a phase I/II study of CTCE-9908, a novel anticancer agent that inhibits CXCR4, in patients with advanced solid cancers. Eur J Cancer. 2008;6:127.
OncoTargets and Therapy

\section{Publish your work in this journal}

OncoTargets and Therapy is an international, peer-reviewed, open access journal focusing on the pathological basis of all cancers, potential targets for therapy and treatment protocols employed to improve the management of cancer patients. The journal also focuses on the impact of management programs and new therapeutic agents and protocols on

\section{Dovepress}

patient perspectives such as quality of life, adherence and satisfaction. The manuscript management system is completely online and includes a very quick and fair peer-review system, which is all easy to use. Visit http://www.dovepress.com/testimonials.php to read real quotes from published authors. 